\title{
COMPUTATION OF EMI FIELDS GENERATED DUE TO CORONA ON HIGH VOLTAGE OVER HEAD POWER TRANSMISSION LINES
}

\author{
S. K. Nayak and M. Joy Thomas \\ Department of High Voltage Engineering \\ Indian Institute of Science, Bangalore 560012
}

\begin{abstract}
Corona generated pulsed currents on high voltage transmission line conductors radiate electromagnetic field, which inturn can interfere with communication systems, radio as well as television receivers operating nearby. In this paper, corona generated electromagnetic interference (EMI) field in $\mathrm{dB}$ at an observation point near the ground has been computed assuming ground as a perfect conductor. It has been observed that the interference field starts abruptly at the corona inception voltage and it increases with the voltage stress on the conductor. It is also observed that the EMI field reduces with increase in lateral distance and also with increase in frequency as well as the conductor diameters for the same line voltage.
\end{abstract}

\section{Introduction}

EMI field or radio noise field from high-voltage transmission lines are caused by corona, which is essentially due to the electrical breakdown of the air surrounding the conductors at higher voltage. When the conductor surface electric field exceeds the corona onset electric field, a partial electrical breakdown occurs in the surrounding air medium near the conductor surface and is called the corona discharge. The increase of conductor surface gradient takes place with increase of supply voltage. In addition, organic contamination or attachment of water droplets also may contribute to localized field enhancement. When organic particles or water droplets are attached to the conductor surface, the charge accumulation at that point increases which enhances the local electric field. The intensification of surface gradient locally leads to the corona discharge.

The streamer generated during corona discharge, transports electric charge into the surrounding air during the discharge cycle. These moving charges contribute directly to the noise fields. 'They also cause currents to be induced on the transmission line conductors. Since the charge is moved by a time varying electric field, it is equivalent to a current pulse and this current pulse is the source of EMI field.

When a communication line passes near the corridor of a HV or EHV transmission line, if the frequency of the radiated EM signal due to corona matches with that of the transmitted signal on the communication line, then the communication signal may get distorted. To mitigate this effect, the communication line should pass at a safe distance away from the transmission line. Hence there is a need to estimate the radiated EMI signal in $\mathrm{dB}$ at a given distance from the HV or EHV transmission line.

In this paper, radiated EMI in $\mathrm{dB}$ is computed for a single conductor high voltage over headline. This theoretical result is compared with the published experimental results available in the literature. In the computational work, earth is considered as an infinitely conducting ground.

\section{Physical description of corona and EMI}

When alternating supply voltage energizes the conductor, the conductor surface electric field exceeds the corona onset electric field of the conductor. The corona discharge occurs in both positive and negative half cycle. So the corona is divided into positive and negative corona depending upon the polarity of the supply voltage.

When the conductor is positive with respect to ground, an electron avalanche moves rapidly into the conductor leaving the heavy positive-ion charge cloud close to conductor, which drifts away. The rapid movements of electrons and motion of positive ions gives the steep front of the pulse, while the further drift of positive ions will give slow tail of the corona pulse.

When conductor is negative with respect to the ground, an electron avalanche moves away from the energized conductor and the positive heavy ions move towards the conductor. Since the heavy positive ions are moving towards the higher electric field, their motion is very rapid which gives rise to a much sharper pulse than the positive pulse. Due to rapid moment of the electrons from the conductor surface, the electric field regains its original value at conductor surface very quickly than in the case of positive polarity. Thus the negative corona pulses are lower in amplitude and lower in rise and fall times as compared to positive corona pulses. They have also higher repetition rates than the positive pulse. These characteristics are listed in table 1.

The corona current pulses thus generated can be represented by a double exponential current waveform which is given as follows

$i(t)=K i_{p} \cdot\left(e^{-\alpha t}-e^{-\beta t}\right), t \geq 0$

where $i_{p}$ is the amplitude of corona current in $\mathrm{mA}$ and 


\begin{tabular}{|l|l|l|l|l|}
\hline Polarity & $\begin{array}{l}\text { Amplitude } \\
\text { of the } \\
\text { typical } \\
\text { corona } \\
\text { current } \\
\text { pulse(mA) }\end{array}$ & $\begin{array}{l}\text { Rise } \\
\text { time } \\
\text { (ns) }\end{array}$ & $\begin{array}{l}\text { Duration } \\
\text { (ns) }\end{array}$ & $\begin{array}{l}\text { Repetition } \\
\text { rate } \\
\text { (pulse/sec) }\end{array}$ \\
\hline$+\mathrm{ve}$ & $10-50$ & 50 & 250 & $1000-5000$ \\
\hline$-\mathrm{ve}$ & $1-10$ & 10 & 100 & $\begin{array}{l}10000- \\
50000\end{array}$ \\
\hline
\end{tabular}

$K, \boldsymbol{\alpha}$ and $\boldsymbol{\beta}$ are constants

The rise time and fall time of the current pulse depends on $\alpha$ and $\beta$ values respectively.

These constants for positive and negative corona are shown in equations $\mathbf{2}$ and $\mathbf{3}$ respectively [1].

The positive corona current can be represented as

$i(t)=2.335 . i_{p} \cdot\left(e^{-0.01 t}-e^{-0.0345}\right)$

and the negative corona current can be represented as

$i(t)=1.33 i_{p}\left(\mathrm{e}^{-0.019}-e^{-0.285 t}\right)$

The frequency domain representation of the current pulse given by equation (1) is as follows

$$
I(s)=\frac{K . i_{p} \cdot(\beta-\alpha)}{(s+\alpha)(s+\beta)}
$$

and $I(j \omega)=\frac{K \cdot i_{p} \cdot(\beta-\alpha)}{\sqrt{-\boldsymbol{\alpha})\left(\boldsymbol{\beta}^{2}+\omega^{2} \mathrm{j}\right.}}$

The positive and negative corona current pulse waveforms in time and frequency domain are shown in figures land 2 respectively. In these waveforms $\boldsymbol{i}_{p}$ is taken as $20 \mathrm{~mA}$ and $5 \mathrm{~mA}$ for positive -and negative corona currents respectively.

The critical frequencies of interest are given by

$f_{\alpha}=\frac{\alpha}{2 \pi}$ and $f_{\beta}=\frac{\mathbf{P}}{2 \pi}$

In case of positive corona,

$f_{\alpha}=1.59 \mathrm{MHz}$ and $f_{\beta}=5.49 \mathrm{MHz}$ and

for negative corona,

$f_{\alpha}=3.02 \mathrm{MHz}$ and $f_{\beta}=45.36 \mathrm{MHz}$

Since the AM broadcast frequency band is in the range of $1.6 \mathrm{MHz}$ and this matches with the frequency of positive corona pulse, positive corona is considered to be a major source of EMI for radio transmission.

\section{Method of calculation}

Figure 3 shows the configuration of high voltage transmission line used in the study. A conductor at a height $\mathrm{h}$ (along the $\mathrm{Y}$-axis) and radius $a$ is assumed to be stressed with a voltage $V$ at power frequency. The observation point $\mathrm{P}$ is assumed to be at a distance $\mathrm{d}$ meters along the $\mathrm{x}$-axis. The earth is assumed to be perfectly conducting and hence to estimate the field at

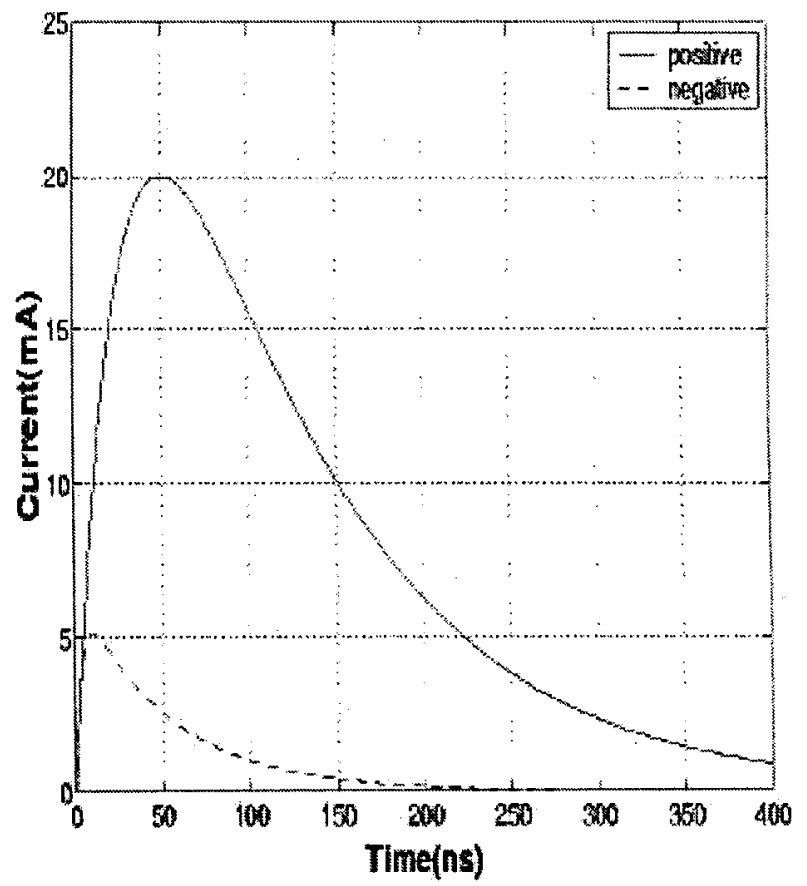

Fig.1: Time domain representation of corona current pulses

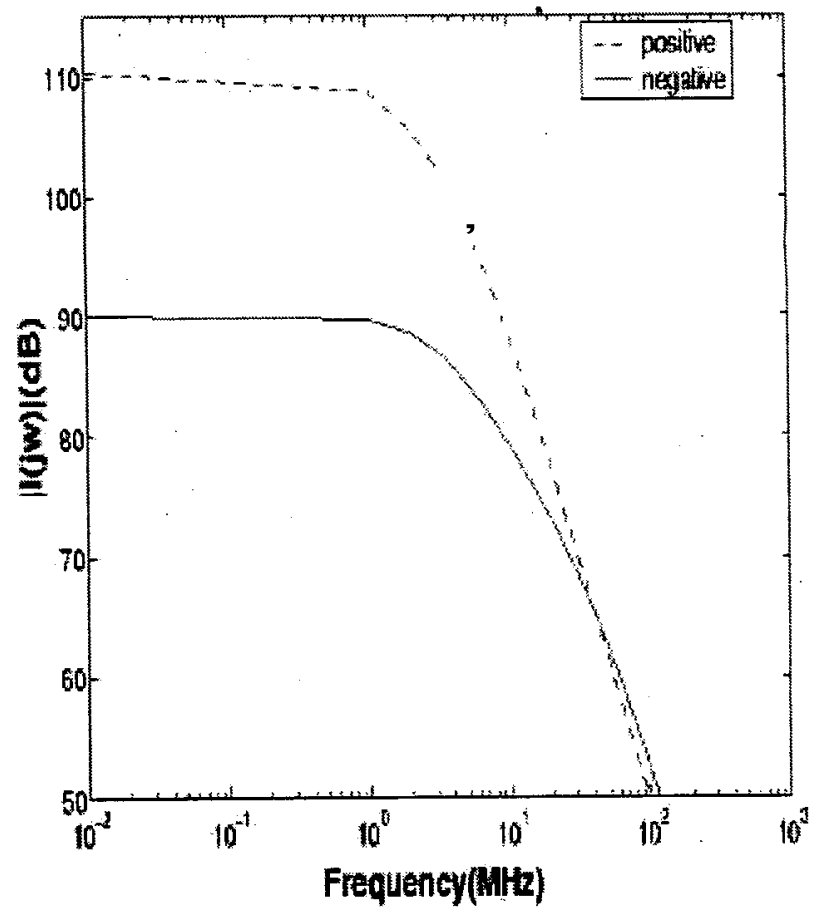

Fig.2: Frequency spectra of corona current pulses 
point $\mathrm{P}$, the contribution of the image conductor is also to be considered. The infinite conductor is parallel to the $\mathrm{Z}$ axis. The electric field $E$ at the surface of the conductor is given by

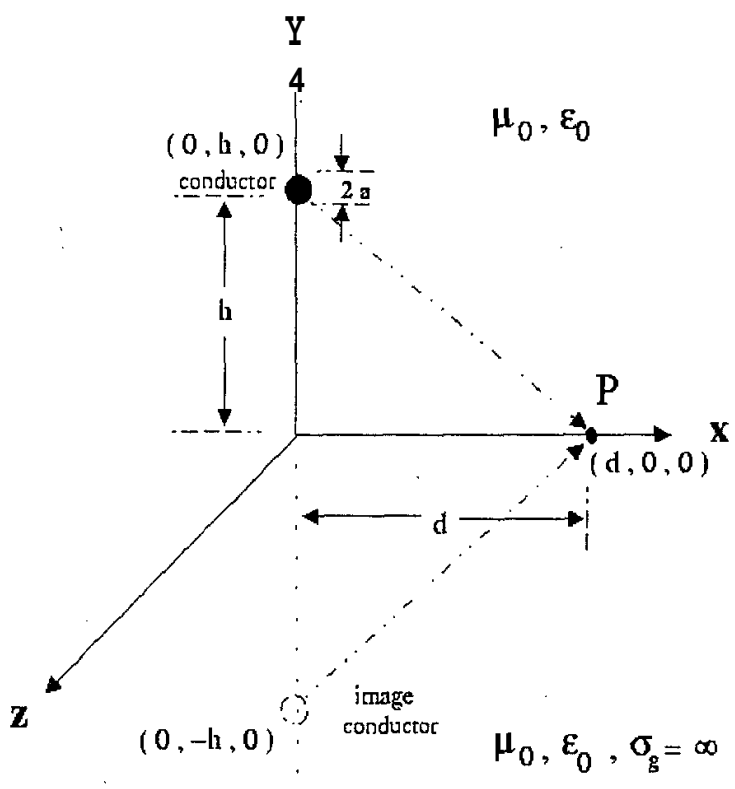

Fig.3: Location of the HV conductor used in the computation of EMI field

$$
E=\frac{V}{a \ln \left(\frac{2 h}{a}\right)}
$$

When the surface gradient due to supply voltage exceeds the onset electric field of the conductor, the corona discharge occurs in the vicinity of the conductor.

The corona onset field $E_{C}$ in $\mathrm{kV} / \mathrm{cm}$ is given by the wellknown Peek's formula [2] which is given as follows

$$
E,=21.1 m \delta\left(1+\frac{0.301}{\sqrt{a \delta}}\right)
$$

where $a$ is conductor radius in $\mathrm{cm}$ and $\mathrm{m}$ is conductor surface irregularity factor which is 1 for ideally smooth and clean conductors and it varies between 0.75 and 0.85 for stranded conductors.

$\delta$ is relative air density factor and is given by [3]

$\boldsymbol{\delta}=\left(\frac{273+t_{0}}{273+t}\right)\left(\frac{p}{p_{o}}\right)$

where $p_{0}$ and $t_{0}$ are standard atmospheric pressure and temperature. $\boldsymbol{p}$ and $\boldsymbol{t}$ are the ambient pressure and temperature for test condition.

The corona current $J$ is given by the equation

$$
J=q \mu(E)^{2}
$$

where $q$ is charge of electron in coulomb and p.n $\mathrm{cm}^{2} / \mathbf{V}-\mathbf{s}$ is mobility of the electron in free space.

The equivalent circuit of an elemental length of overhead HV line with uniform corona current

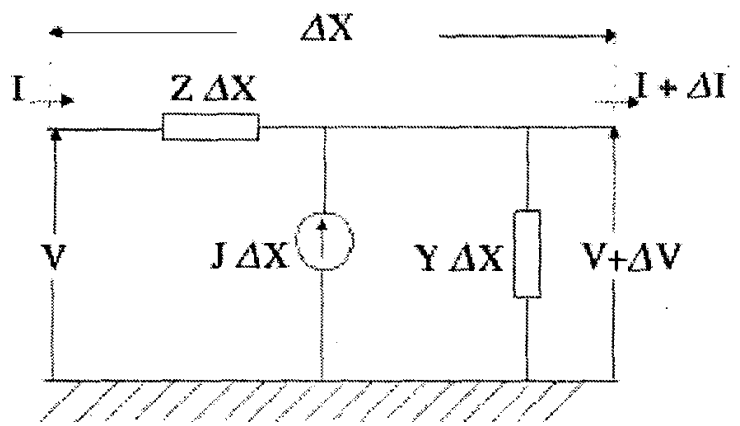

Fig.4: Equivalent circuit for an elemental length of the overhead HV line

injection of $J \mathrm{~A} / \sqrt{m}$ per unit length is shown in figure 4 . The following differential equations describe the current voltage relations of the overhead line. Since the corona current injection is in the form of pulse trains, $J$, and therefore $V$ and $I$ are rms values at a given frequency.

$$
\begin{aligned}
& \frac{d V}{d x}=-Z I \\
& \frac{d I}{d x}=-Y V+J
\end{aligned}
$$

The parameters $Z$ and $Y$ are the series impedance and shunt admittance respectively per unit length of the line at same the frequency.

When this corona current $J$ is injected at a particular point on the transmission line, the current is equally divided and propagates in the both directions. Therefore, the corona induced current $I$ is given by

$I=\frac{J}{2 \sqrt{Y Z}}$

where

$Y=$ admittance of the over head transmission line

$Z$ =impedance of the over head transmission line

The magnetic field $\mathrm{H}_{X}$ at point $\mathrm{P}$ due to the conductor and its image is

$\mathrm{H}_{X}=\frac{I h}{\pi\left(d^{2}+h^{2}\right)}$

The radiated electric field $E_{Y}$ at point $\mathbf{P}$ is given by

$\mathrm{E},=\mathrm{Z}_{0} \mathrm{H}_{X}$

where $\mathbf{Z}_{0}$ is the wave impedance in free space and is

given by $Z_{0}=\sqrt{\frac{\mu_{0}}{\varepsilon_{0}}}$ 
Hence $\mathrm{E}_{Y}=120 \pi \mathrm{H}_{X}$

The EMI field in $\mathrm{dB}$ with reference to $1 \mu \mathrm{V} / \mathrm{m}$ is given

by $E_{Y}=20 \log _{10}\left[\frac{E_{y}(\mu V / m)}{1(\mu V / m)}\right]$

\subsection{Results and Discussions}

A conductor at a height of $15 \mathrm{~m}$ above the ground and diameter of $3 \mathrm{~cm}$ has been chosen for the study. The point of interest is located on ground at a distance of $15 \mathrm{~m}$ laterally from the conductor. The conductor voltage has been varied from $290 \mathrm{kV}$ to $590 \mathrm{kV}$ and EMI field, computed. The results are presented in the following sections.

\subsection{Variation of EMI field with conductor voltage :}

When the supply voltage exceeds the corona inception voltage of the conductor, the EMI field increases rapidly with the voltage. Figure 5 shows the variation of EMI

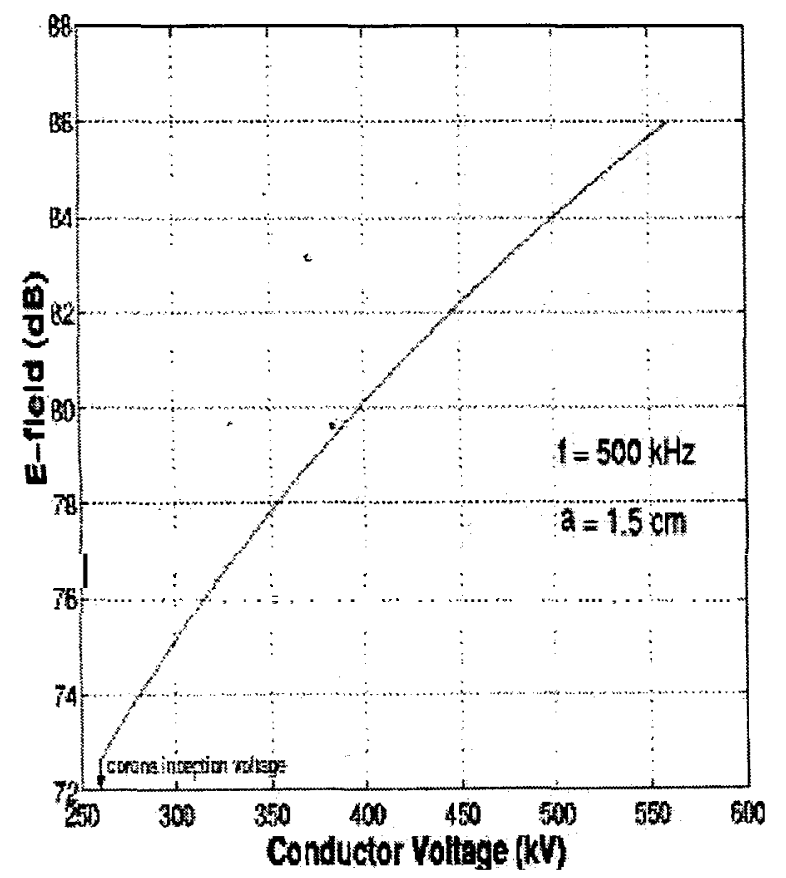

Fig.5: EMI field variation with conductor voltage

field in $\mathrm{dB}$ with the conductor voltage.

\subsection{Variation of EMI field with lateral distance:}

The lateral profile describes how the noise level falls off with increasing distance from the line. When the distance from the conductor increases laterally the EMI field decreases rapidly as shown in figure 6 . The difference of noise level of present result and CIGRE result is almost $4.8 \mathrm{~dB}$ at $5 \mathrm{~m}$ and $10.1 \mathrm{~dB}$ at $40 \mathrm{~m}$ lateral distances where as the noise level of both are $74 \mathrm{~dB}$ at $16 \mathrm{~m}$ distance. This variation could be due to the approximate formula used in the CIGRE work.

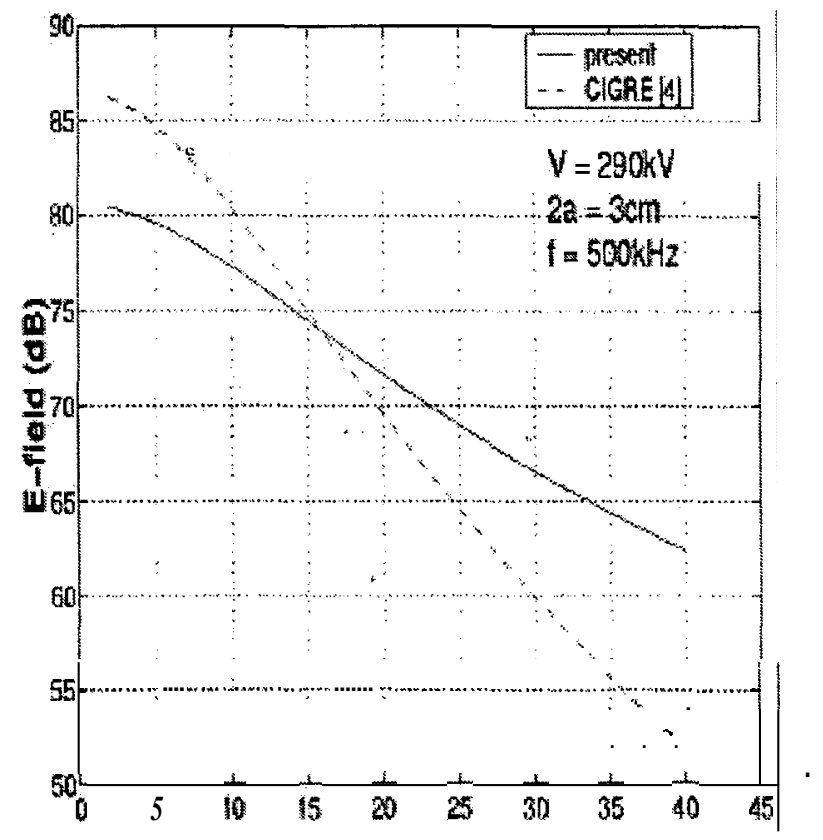

\subsection{Variation of EMI field with conductor height}

Figure 7 shows the variation of EMI fields for conductor heights of $15 \mathrm{~m}, 17.5 \mathrm{~m}$ and $20 \mathrm{~m}$. In general, as the height increases, the EMI level decreases. But after $a^{\text {a }}$ certain lateral distance, the difference in EMI level for different heights shows a reverse trend.

\subsection{Variation of EMI field with frequency.}

Most of the measurements in general are made at one frequency as it is too difficult to measure at all the frequencies. At site $0.2 \mathrm{MHz}$ and in the laboratory 0.5 or $1 \mathrm{MHz}$ frequencies are used for the measurement of EMI. Computation (figure 8) shows that the EMI (in $\mathrm{dB}$ ) from the conductor decreases with increase in frequency.

\subsection{Variation of EMI field with conductor diameter}

When conductor diameter increases, the EMI field decreases with lateral distance. Figure 9 illustrates the EMI $(\mathrm{dB})$ variation with lateral distance for different diameters of the conductor. 


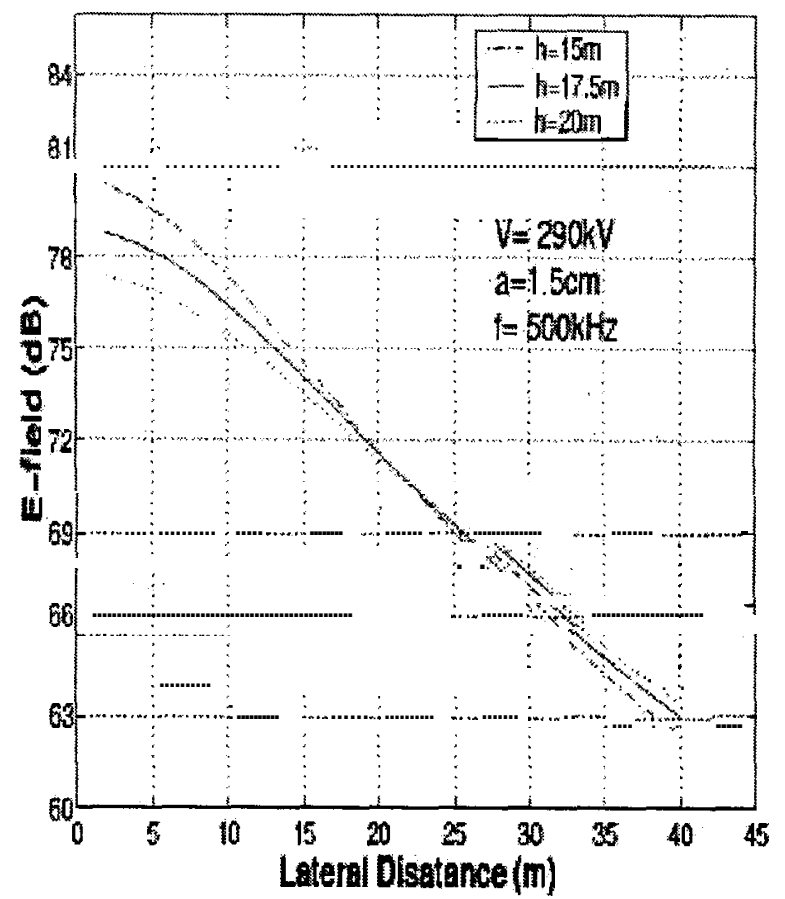

Fig.7: EMI field variation with height of the conductor

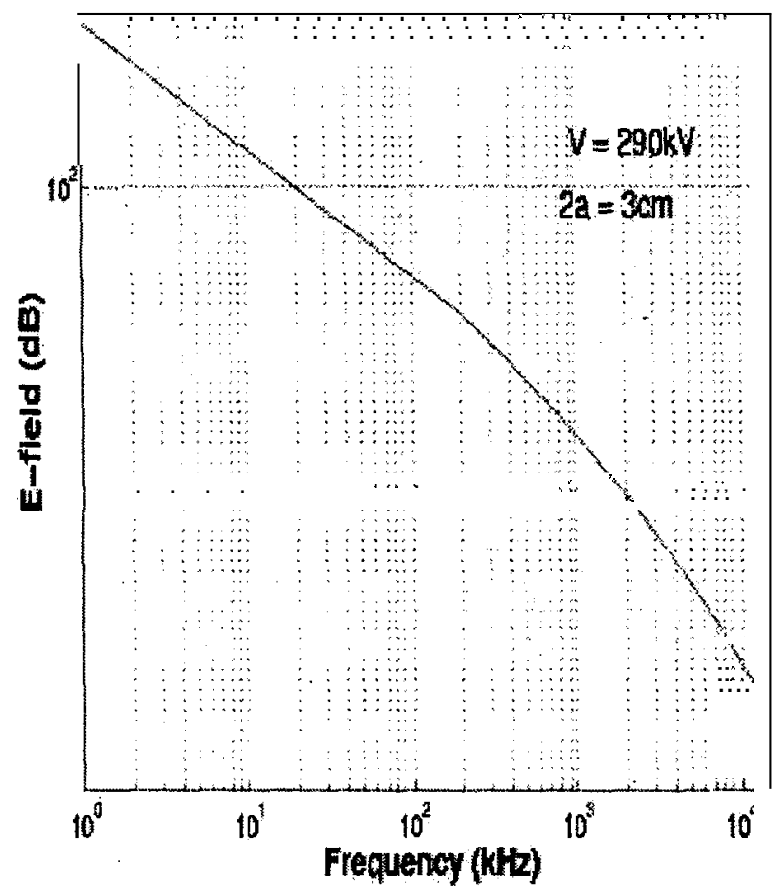

Fig.8: EMI field variation with frequency

\section{Conclusion:}

EMI field due to corona generated pulse current has been computed for differian; conductor voltages, lateral observation points, heignt of the cinductor above ground as well as conductor diameters. It has been observed that the EMI field abruptly starts at the corona inception voltage and increases with the voltage stress on the conductor and decreases with lateral distance. The EMI field in general decreases with conductor height, frequency and diameter

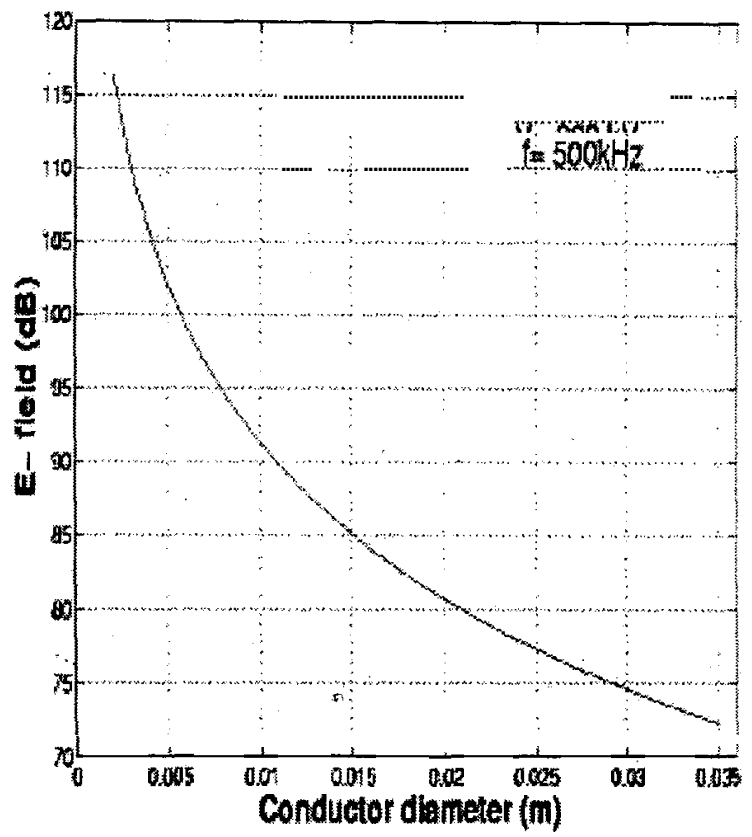

Fig.9: EMI field variation with conductor diameter

\section{Acknowledgement}

The authors thank the authorities of the Indian Institute of Science for the permission to publish this paper.

\section{REFERENCE}

1. P. Sarma, Maruvada, "Corona Performance of HighVoltage Transmission Lines", Research Studies Press Ltd., England, April, 2000, P 114.

2. F. W. Peek, "Dielectric phenomena in High Voltage Engineering" $3^{\text {rd }}$ edition, New York, Wiely, 1929 P 81.

3. E. Kuffel, W.S. Zaengl, "High Voltage Engineering Fundamentals", Pergamon Press, 1st edition, New York, 1984,P 99.

4. Robert G. Olsen, Steven D. Schennum, Vernen L. Chartier "Comparison of several methods for calculating power line electromagnetic interference levels and caibration with long term data", IEEE Transactions on power Delivery, Vol. 7, No. 2, April 1992,pp 903-912. 